Lexis Vol. XLV (1) 2021: 191-225

\title{
La consideración y tratamiento del error en la producción lingüística de aprendices de segundas lenguas: una aproximación teórica
}

\author{
Belén C. Muñoz Muñoz \\ https://orcid.org/0000-0001-8350-7250 \\ Universidad Católica de la Santísima Concepción \\ bmunoz@ucsc.cl
}

\section{RESUMEN}

El error y su corrección en el proceso de aprendizaje de una segunda lengua continúa siendo un tema de debate entre los especialistas del área y los encargados de desarrollar los procesos de instrucción. Cuestiones como qué constituye un error, la pertinencia de la corrección, la efectividad de las distintas técnicas que permiten implementar un tratamiento correctivo, entre otras, surgen como interrogantes que requieren ser analizadas. Así, esta minuciosa revisión teórica emerge como una contribución tanto al trabajo investigativo acerca de la corrección del error como a la toma informada de decisiones en un contexto de enseñanza-aprendizaje de una segunda lengua, sustentando, desde un punto de vista teórico, posibles acciones empíricas tendientes a mejorar los procesos de adquisición lingüística.

Palabras claves: errores en segunda lengua, adquisición lingüística, tratamiento del error, proficiencia lingüística 
Consideration of Error and its Treatment in Second Language Learners Production: A Theoretical Approach

\begin{abstract}
Error and its correction in the process of second language learning continues to be a topic of debate among specialists in the area and among those in charge of carrying out the instructional processes. Issues such as what constitutes an error, the appropriateness of correction, the effectiveness of the different techniques that allow to implement a corrective treatment, among others, arise as inquiries that need to be analyzed. Thus, this detailed theoretical review emerges as a contribution to both the research work on error correction and the informed decision-making in a teaching-learning context of a second language, supporting, from a theoretical point of view, potential empirical actions tending to improve the acquisition process.

Keywords: errors in second language, acquisition, error treatment, language proficiency
\end{abstract}

\title{
1. Introducción
}

La corrección de errores en un contexto de aprendizaje de una nueva lengua constituye un proceso que ha acaparado la atención de diversos estudiosos en el ámbito de la Lingüística Aplicada, puesto que representa un elemento irresoluto a pesar del desarrollo investigativo. La consideración respecto de la importancia de corregir los errores que naturalmente se producen en el proceso de adquisición lingüística ha sido oscilante a lo largo de los años. En primera instancia, la necesidad de definir qué corresponde a un error originó diversas discusiones entre los teóricos, quienes han buscado determinar con certeza cuáles son aquellas desviaciones que pudiesen obstaculizar el aprendizaje de la lengua en estudio. Ya en 1967, Corder distinguía entre dos tipos de errores: sistemáticos y no sistemáticos; siendo los primeros una desviación de la lengua producto del desconocimiento de la regla y los segundos, una falta cometida por un lapsus. Si bien en un principio se consideraba imperioso 
aminorar o evitar los errores cometidos por los aprendices, desde la década del 70 los errores se reconocen como una unidad fundamental para conocer los procesos de adquisición lingüística y para llevar a cabo la comprobación de hipótesis que suscita el aprendizaje (Corder 1981).

Ahora bien, en búsqueda de promover el proceso de adquisición, se ha señalado que es fundamental corregir no solo los errores sistemáticos sino también aquellas faltas espontáneas, pues de esta manera se evita la fosilización de las imprecisiones, asegurando esto la exactitud lingüística (v. Hendrickson 1978, Sheen 2011, Bitchener y Ferris 2012). No obstante, la corrección del error ha sido una tarea compleja que no ha estado exenta de cuestionamiento en cuanto a su efectividad (Truscott 2004, 2007, 2009; Suzuki, Nassaji y Sato 2019) y, a pesar de la gran variedad de estudios que se ha llevado a cabo, aún no es posible proporcionar respuestas concluyentes al respecto pues los resultados obtenidos son poco generalizables. Sin embargo, especialmente en un escenario de instrucción formal, existe cierto consenso en cuanto a la necesidad de proporcionar retroalimentación a quienes aprenden una segunda lengua con el propósito de automatizar un uso preciso de las estructuras léxicogramaticales del nuevo código. Sumado a esto, se ha comprobado que los aprendices consideran fundamental ser corregidos durante su proceso de aprendizaje (Brown 2009; Jean y Simard 2011; Martínez 2013; Schulz 1996, 2001; Guénette 2012). Es más, Lyster, Saito y Sato (2013: 8) sostienen que "the extent to wish learners want to be corrected is generally greater than teachers' wish to provide correction".

En este escenario, se vuelve fundamental analizar las sugerencias teóricas respecto de los errores en la adquisición de lenguas, la implicancia de un tratamiento correctivo, las estrategias de corrección pertinentes, el lugar de la corrección de acuerdo con las teorías de adquisición, entre otras cosas, puesto que representan aspectos significativos para los investigadores del área y para quienes están a cargo de llevar a cabo un proceso de instrucción lingüística de una lengua adicional. 


\section{Revisión teórica}

2.1. Los errores en la adquisición de segundas lenguas

El tratamiento del error, en especial, en contextos de instrucción de lenguas, es una cuestión que no está del todo resuelta. Diversas teorías acerca de la adquisición de segundas lenguas (ASL) difieren en el impacto que la corrección del error tiene en la promoción de la interlengua (IL) de los aprendices. Hay quienes sostienen que la corrección de los errores facilita la adquisición o aprendizaje de la lengua en estudio (Long 1996, Sheen 2010, Bitchener y Ferris 2012, Ortiz 2016, Muñoz 2017) y quienes señalan que este procedimiento es ineficaz, e incluso perjudicial, por lo que debería ser abandonado (Truscott 2004, 2007, 2009). En contra de esta postura, resulta fundamental manifestar que los aprendices de una lengua adicional, en específico aquellos con niveles básicos de proficiencia, requieren la orientación de sus profesores para superar el desconocimiento léxico y morfosintáctico propio de un proceso de aprendizaje y, de esta manera, potenciar la construcción de su IL y desarrollar estrategias que les permitan identificar, corregir y evitar sus imprecisiones.

En este contexto, se torna fundamental establecer qué constituye un error, aspecto que ha sido abordado en el marco de diversas investigaciones en ASL. Ellis (1994) señala que un error corresponde a una realización no deseada de la lengua, tanto en lo comprensivo como en lo productivo. En tanto, Ferris (2011) explica que un error es una forma morfológica, sintáctica y/o léxica que se desvía de la regla de la lengua meta. Corder (1967) distingue entre errores sistemáticos y no sistemáticos o, en palabras de Brown (2007), errors y mistakes, respectivamente. Los errores sistemáticos se relacionan con el nivel de competencia lingüística del estudiante, es decir, con un conocimiento restringido de las reglas de la lengua; por su parte, los errores no sistemáticos o mistakes son faltas que emergen en el proceso de uso comunicativo del nuevo código e incluso en el uso de la lengua materna, debido a algún estado físico o sicológico en particular. Si bien al analizar estas definiciones pareciera bastante sencillo, determinar qué es un error se torna un procedimiento 
complejo en la medida en que se atienden las especificidades propuestas por otros autores.

En el siglo XX los errores o desviaciones sistemáticos constituían elementos indeseados que debían ser reducidos y/o eliminados por quienes estaban a cargo del proceso de instrucción (Bitchener y Ferris 2012); sin embargo, este juicio sufrió ciertos cambios durante la década de los 70, periodo en el que las imprecisiones lingüísticas pasaron a considerarse unidades significativas en el proceso de adquisición de una L2, ya que entregan información acerca del modo en que se adquiere o aprende una lengua, del estado y evolución de dicho aprendizaje y, fundamentalmente, permiten a los estudiantes comprobar sus hipótesis sobre la naturaleza de la lengua, dando lugar a la adquisición (Corder 1981).

En esta misma época, distintos investigadores comenzaron a analizar los errores de competencia o sistemáticos intentando explicarlos. Así, Burt (1975) los clasificó como “errores globales”, que corresponden a imprecisiones léxicas que perjudican la comprensión de los enunciados léxicos que interfieren y "errores locales", que son desviaciones morfológicas que no tienen injerencia en la compresión del mensaje.

Ahora bien, a partir de las diferenciaciones expuestas surge la disyuntiva de determinar si todos los errores merecen ser corregidos y si todos se beneficiaban de dicha corrección. Así, en el ámbito pedagógico Hendrickson (1978) recomienda corregir los errores globales porque son estos los que obstaculizan una comunicación efectiva. Contrariamente, Sheen (2011), y Bitchener y Ferris (2012) destacan que no solo es necesario reparar los errores periódicos, sino que también aquellos errores que, sin perjudicar la comprensión del mensaje, estigmatizan a quien produce el enunciado incorrecto. Estas apreciaciones conforman una materia que genera gran interés e inquietud en ambientes de enseñanza de lenguas, puesto que se vuelve esencial identificar y clasificar las desviaciones lingüísticas de los aprendices para atenderlos e impedir su fosilización. Por su parte, Suzuki, Nassaji y Sato (2019) sostienen que el tipo de error tratado mediaría la efectividad y durabilidad de la corrección debido 
a que no todas las desviaciones responden de la misma manera a un tratamiento correctivo. En este escenario, desarrollar técnicas apropiadas para corregir tales faltas y resguardar la precisión lingüística parece ser una necesidad sustancial. Por ello, el tratamiento del error se ha convertido en un área considerablemente estudiada con el propósito de identificar aquellas imprecisiones que se benefician en mayor medida de un procedimiento como este y delimitar las técnicas correctivas más efectivas al momento de llevar a cabo un tratamiento correctivo. No obstante, a pesar de este desarrollo investigativo, persisten muchas interrogantes al respecto, cuestión que demanda profundizar en la literatura y en la evidencia empírica existente.

\subsection{El tratamiento del error}

El tratamiento del error, conocido también como feedback correctivo (FC), constituye una tarea dificultosa a causa de la multiplicidad de elementos que deben tenerse en consideración al momento de responder a las imprecisiones de los aprendices. Diversas interrogantes respecto a este tema fueron introducidas hace más de 40 años por Hendrickson (1978) y, a pesar del paso del tiempo y la diversidad de investigaciones al respecto, parecen lejos de estar resueltos (Lyster y Ranta 1997, Sheen y Ellis 2011). Estos cuestionamientos pueden plantearse del siguiente modo:

1. ¿Se deben corregir los errores de los estudiantes?

2. ¿Cuándo se deben corregir los errores de los estudiantes?

3. ¿Qué errores se deben corregir?

4. ¿Cómo se deben corregir tales errores?

5. ¿Quién debe corregir los errores de los estudiantes?

Precisamente, es fundamental reflexionar respecto de esta problemática, pues siguen constituyendo una materia central en la discusión teórica acerca del FC (v. Sheen 2011, Bitchener y Ferris 2012, Pawlak 2014, Li 2017). 
2.2.1. ¿Se deben corregir los errores de los estudiantes?

Si bien en un comienzo, la corrección de errores se fundamentaba en las razones para corregirlos y no en el beneficio o el papel que cumplía tal corrección en la adquisición de una L2 (Bitchener y Ferris 2012), recientemente se ha admitido que la corrección es efectiva para promover la IL de los aprendices (Sheen 2011, Bitchener 2012), puesto que esto facilitaría a los estudiantes identificar sus imprecisiones, comprobar sus hipótesis de la lengua meta y descubrir las funciones y limitaciones de las estructuras léxico-gramaticales de la lengua en estudio (Corder 1973, Sheen 2011). Otro aspecto que valida el tratamiento del error corresponde a la necesidad y a la voluntad de los aprendices de una L2 de ser corregidos, tal como se ha demostrado en distintas investigaciones (Ferris 1999, 2004; Hyland y Hyland 2006; Sheen 2006, 2007).

A pesar de estos sólidos argumentos en favor de la corrección de errores, Truscott (1996) es enfático en señalar que reparar las imprecisiones de los aprendices no tiene un impacto significativo en la adquisición del nuevo código lingüístico, puesto que, si el tratamiento correctivo tuviese alguna efectividad, sería solo a nivel de conocimiento consciente o explícito; es decir, el conocimiento inconsciente o implícito, necesario para el uso en línea de la lengua, no sería desarrollado. No obstante, no es sencillo aprobar o refutar esta hipótesis debido a que el estudio de procesos cognitivos no es una tarea sencilla. Probablemente, esta sea la razón por la que "the implicit/explicit issue lies at the heart of the debate about the efficacy of error correction" (Shintani y Ellis 2013: 287).

\subsection{2. ¿Cuándo se deben corregir los errores de los estudiantes?}

Esta pregunta representa una inquietud fundamental en el ámbito del FC debido a la inexistencia de un criterio común respecto de si los errores deben corregirse de manera inmediata o diferida (Sheen 2011). En la búsqueda de respuestas taxativas, se ha sugerido que la variable temporal en un tratamiento del error puede abordarse desde dos perspectivas complementarias: una psicolingüística y una pedagógica (Lightbown 1998). 
Desde un punto de vista psicolingüístico, se indica que la corrección del error es beneficiosa cuando apunta a la etapa correcta de desarrollo de adquisición (Pienemann y Keßler 2012). Ahora bien, es necesario considerar también que los resultados de investigaciones en esta área parecen no ser del todo concluyentes, pues se ha sugerido que la entrega de FC puede ser beneficiosa incluso cuando está dirigida a formas lingüísticas allende el desarrollo real de los sujetos. Por ello, se necesita de más y variados estudios empíricos que permitan elaborar respuestas concluyentes a este respecto.

Desde una perspectiva pedagógica, se recomienda corregir los errores atendiendo a las consideraciones incluidas en la planificación instruccional; sin embargo, parece no existir suficiente acuerdo en torno a la efectividad de una corrección integrada al proceso de instrucción o de una aislada de este (Spada 2011).

Ahora bien, independientemente del cuándo llevar a cabo la corrección, se destaca la necesidad de integrarla en las actividades comunicativas, pues según la evidencia acerca del aprendizaje y la memoria, los estudiantes recuerdan mejor cuando evocan el contexto en el que ocurrió el aprendizaje (Lightbown 1998).

A pesar de la claridad de las orientaciones descritas, surge la interrogante respecto de si todos los errores responden de la misma manera a la corrección, o bien, cuáles son los que se beneficiarían en mayor medida de los mecanismos correctivos.

\subsection{3. ¿Qué errores se deben corregir?}

Identificar las estructuras lingüísticas cuya adquisición se ve favorecida por un tratamiento correctivo es una tarea compleja, puesto que no existe claridad acerca de la complejidad de las reglas léxicogramaticales de la L2. La determinación respecto de qué aspectos hacen que una regla sea simple o compleja de adquirir y cuál de ellas se favorece de una instancia correctiva es un asunto ciertamente problemático, cuestión que se acentúa en el campo de la morfosintaxis. DeKeyser (2009: 58) apunta a la distinción de las "reglas que son fáciles de adquirir, pero difíciles de aprender y reglas que son fáciles de aprender, pero difíciles de adquirir”, siendo estas últimas 
aspirantes prioritarias para una instrucción focalizada. Por su parte, Harley (1993) señala que existen cuatro características propias de aquellas formas que responden de manera positiva a un tratamiento correctivo. A saber:

1. Difieren de modo no evidente de la L1.

2. Son irregulares, poco frecuentes o carecen de relevancia perceptiva en el input.

3. Son importantes para el desarrollo de una comunicación efectiva.

4. Pueden ser malinterpretadas.

Así, en el intento por clasificar las estructuras de la lengua y especificar aquellos errores que responden efectivamente a un tratamiento correctivo, Ferris $(1999,2011)$ caracteriza las imprecisiones como errores "tratables" y "no tratables", siendo los primeros los que responderían de mejor forma a un tratamiento correctivo. No obstante, establecer qué estructuras son fáciles de aprender y difíciles de adquirir o cuáles son tratables no es una tarea simple (Sheen 2011). Es más, aun cuando ciertas estructuras siguen determinados patrones para su configuración, fenómeno que podría indicar cierta simplicidad de la regla, las características propias de dicha estructuración pueden variar en cuanto a su complejidad, obstaculizando así una clasificación efectiva del error. A este respecto, Ellis (2009a: 6) es categórico en afirmar que "ninguno de estos criterios es fácil de implementar en la práctica”, idea compartida por Pawlak (2014: 123), quien sostiene que "most of these recommendations are unfeasible or at a very least extremely difficult to implement". De este modo, aspectos como la discrepancia en cuanto a la gravedad del error, la inexistencia de una teoría extensamente aceptada acerca de la complejidad de las reglas que fundamente una decisión acabada respecto de los errores tratables y, en el ámbito escolar, la falta de tiempo de los docentes para determinar cuáles son las estructuras problemáticas, dificultan una óptima toma de decisiones (Ellis 2009a). En palabras de Truscott (2001: 94) "the selection process should be based primarily on the possibility of success, rather than on perceived need". 
Otro problema que surge corresponde al modo en que deben atenderse las imprecisiones que emergen, de manera natural, en el proceso de incorporación de un nuevo sistema lingüístico; esto es, qué estrategias adoptar en el marco de la acción correctiva.

2.2.4. ¿Cómo se deben corregir los errores de los estudiantes?

La pertinencia de las técnicas correctivas en los distintos escenarios y para las distintas estructuras atendidas permanece siendo una temática de gran debate teórico y empírico. En un intento por sistematizar las estrategias correctivas, han surgido diversas tipologías que permiten al profesor o investigador abordar con rigurosidad el tratamiento del error. De acuerdo con Ellis (2009b: 97), estas clasificaciones constituyen "a basis for a systematic approach to investigating the effects of written corrective feedback". A modo general, tales técnicas correctivas pueden clasificarse en dos grandes grupos: 1) estrategias directas, que involucran reparar el error proporcionando la forma correcta, y 2) estrategias indirectas, que destacan la existencia de alguna imprecisión sin proporcionar la respuesta. Estas a su vez pueden combinarse con otros elementos como, por ejemplo, clarificaciones metalingüísticas, dando paso a técnicas correctivas complementarias (v. Ellis 2009b, Sheen 2011).

Otra dicotomía compete al foco que adopta el tratamiento del error, el que puede apuntar a la corrección de todos los errores producidos por el estudiante, FC no focalizado o extensivo, o al tratamiento sistemático de un número limitado de estructuras, FC focalizado o intensivo. Si bien ambos tipos de tratamiento han mostrado resultados favorecedores en el proceso de aprendizaje, el FC focalizado ha demostrado ser muy efectivo en el mejoramiento de la precisión gramatical de determinadas estructuras (v. Bitchener, Young y Cameron 2005; Bitchener 2008; Bitchener y Knoch 2009; Sheen, Wright y Moldawa 2009; Shintani, Ellis y Suzuki 2014; Muñoz 2017; Muñoz y Ferreira 2017).

No obstante, a pesar del número y variedad de estudios tendientes a identificar las estrategias más efectivas, se observa que los hallazgos son más bien discordantes y poco generalizables pues se circunscriben a contextos de instrucción determinados. 
2.2.5. ¿Quién debe corregir los errores de los estudiantes?

Bitchener y Ferris (2012) sostienen que la delimitación del agente idóneo para llevar a cabo la corrección del error constituye una interrogante que solo puede ser respondida desde la intuición debido a la escasa evidencia empírica existente. Sin embargo, diversos investigadores indican que el tratamiento del error tiene una significancia mayor para la modificación de la IL cuando es proporcionada por los profesores y que los estudiantes prefieren ser corregidos por estos, puesto que sus reparaciones son más precisas y confiables ya que poseen un nivel de competencia lingüística superior (Amrhein y Nassaji 2010, Ghani y Asgher 2012).

Otra práctica correctiva que ha mostrado ser beneficiosa corresponde a la retroalimentación proporcionada por pares. Distintos estudios proponen que tal corrección tiene efectos favorables en la promoción del aprendizaje y debería considerarse como una estrategia significativa en la corrección del error (Villamil y De Guerrero 1998, Berg 1999, Rollinson 2005). Por el contrario, existen estudios que sugieren que los estudiantes muestran cierta reserva en cuanto a la calidad de este tipo de corrección, puesto que no confían del todo en ella (Miao, Badger y Zhen 2006).

Pues bien, hasta ahora se ha realizado una revisión sustancial respecto del papel del error y los cuestionamientos existentes respecto de su corrección en contextos de enseñanza de una L2. Aun cuando la evidencia existente hasta el momento no ha permitido levantar conclusiones taxativas al respecto y los resultados de las investigaciones han sido oscilantes, se destaca la necesidad de abordar las imprecisiones de los estudiantes para potenciar el desarrollo de la procedimentalización y automatización de la lengua en estudio, en especial, en contextos de instrucción formal donde los profesores y los aprendices otorgan un papel relevante a la corrección, en el entendido de que esta técnica promueve el proceso de adquisición lingüística. Por esta razón, se vuelve esencial analizar qué se entiende por FC y cuáles son sus particularidades. 
2.3. Delimitando el concepto de feedback correctivo

Bajo la consideración de que la corrección del error es necesaria y efectiva para el desarrollo lingüístico de los estudiantes de una L2, es fundamental precisar qué es el FC, cuáles son sus posibles realizaciones, cuán efectivo es para la promoción de la precisión gramatical, entre otros aspectos. Por ello, antes de profundizar en la noción de FC, se abordará el término feedback como concepto general para luego introducir los tipos de feedback existentes y sus distintas modalidades. Una vez abarcadas estas conceptualizaciones, se profundizará en aquellas estrategias correctivas que han concentrado en mayor medida la atención de los investigadores.

\subsubsection{El feedback}

En el ámbito de ASL, el feedback es entendido como la información que se proporciona a los estudiantes para que estos puedan revisar su IL (Ellis 1994). Por su parte, Crystal (2008) indica que el feedback corresponde a un proceso a través del cual quien produce un mensaje obtiene, por parte del receptor, una respuesta que le permite verificar la precisión o desviación de su enunciado. Hattie y Timperley (2007) rescatan ciertos elementos de las definiciones anteriores y especifican algunos de los agentes responsables de proporcionar la retroalimentación. Para ellos el feedback es la información acerca de la propia actuación o comprensión, la que puede ser entregada por el profesor, por un libro, por un par, etc. Esta información, respuesta o reacción puede apuntar a cualquier aspecto de la lengua; sin embargo, el vocabulario, la pronunciación y la gramática han sido mayormente considerados (Loewen 2012).

En contextos de enseñanza de lenguas, se ha demostrado que el feedback es un elemento que se presenta en forma natural (Polio 2012, Loewen 2012) y que los profesores son sistemáticos en cuanto a la utilización de algunas estrategias que permiten proporcionar la retroalimentación frente a respuestas correctas o incorrectas de los estudiantes. En este sentido, es posible afirmar que el feedback representa una técnica que sustenta el aprendizaje de una lengua adicional. 
En tal escenario, resulta fundamental precisar que el feedback puede proporcionarse tanto para destacar una realización precisa por parte del estudiante (feedback positivo) o para corregir o indicar alguna imprecisión (feedback negativo).

\subsubsection{Feedback positivo y negativo}

Como ya fue señalado, el feedback puede ser positivo o negativo (Long 1996, Ellis 2009a). El primero ratifica un uso correcto de la lengua por parte del usuario, acto que confirma el avance obtenido en la adquisición de la L2 y fomenta la motivación hacia el aprendizaje, siendo este último un elemento relevante en cualquier proceso de instrucción de un nuevo código lingüístico (Ellis 2006, 2009a; Ushioda y Dörnyei 2012). En tanto, el feedback negativo revela un uso impreciso de la lengua (Sheen 2011); es decir, tiene un propósito correctivo (Ellis 2009a). Este último tipo de feedback, negativo o correctivo, es el que ha llamado la atención tanto de investigadores y de profesores de lenguas que buscan fomentar el uso preciso de una L2 y su adquisición; razón por la cual este tipo de corrección ha sido abordado en mayor profundidad (Sheen 2010, Van Beuningen 2010, Sarandi 2016).

\subsubsection{El feedback correctivo}

El feedback correctivo, llamado también corrección del error, feedback negativo o input negativo (Lochtman 2002, Loewen 2012) ha suscitado el interés de diversos investigadores en el área de ASL y de profesores de idiomas, quienes han intentado demostrar su (in) efectividad en un escenario de instrucción lingüística (Sheen 2010, Van Beuningen 2010, Sarandi 2016). Este interés ha posibilitado la construcción de múltiples definiciones de esta técnica correctiva, siendo una de las primeras la introducida por Chaudron (1977, 1988), quien señala que el FC corresponde a la reacción del profesor tendiente a modificar o desaprobar algún error cometido por un estudiante. Esta descripción es compartida por Lightbown y Spada (2006), quienes además destacan que no solo el profesor es el encargado de realizar las observaciones pertinentes, sino también algún hablante nativo o no nativo. Así, es posible deducir que el FC es 
una estrategia que puede utilizarse tanto en espacios de instrucción formal como en ambientes naturales de aprendizaje.

Ellis, Loewen y Erlam (2006) y Ellis (2006, 2009a) concuerdan en que el FC constituye una respuesta frente a un enunciado errado del aprendiz, idea con la que también coincide Van Beuningen (2010), quien, además, destaca que la significación del FC radica en su peculiaridad de provocar la focalización de la atención de los estudiantes en la forma. En palabras de Sheen (2011: 1), el FC es "una movida del profesor que invita a los estudiantes a prestar atención a la precisión gramatical de algo que han dicho o escrito”.

$\mathrm{Al}$ analizar esta última definición, es posible identificar un elemento que no ha sido abordado por las definiciones hasta aquí presentadas. Dicho componente corresponde a las modalidades en las que se puede proporcionar la evidencia negativa; a saber, en forma oral o escrita. Ello permite entender que el FC corresponde a una estrategia correctiva que puede utilizarse en las distintas habilidades de uso productivo de la lengua en estudio. Precisamente, estos tipos de feedback son los que se abordan a continuación.

\subsubsection{El feedback correctivo oral}

Sheen (2011) establece que el feedback oral corresponde a la corrección on line de los errores de los estudiantes, la que, por lo general, se lleva a cabo de manera inmediata después de producida la imprecisión; es decir, posee un carácter reactivo. Lochtman (2002) sostiene que, desde un punto de vista psicolingüístico, este tipo de corrección promueve que los estudiantes realicen comparaciones cognitivas inmediatas entre sus desviaciones lingüísticas y la lengua meta, procedimiento que promueve la IL de los aprendices.

El FC oral puede proporcionarse en forma implícita o explícita (v. Fig. 1). Cuando el feedback es implícito, no existe una indicación expresa acerca de la producción de algún error y demanda la auto-reparación por parte del estudiante, quien debe notar la provisión de evidencia negativa. Algunas de las estrategias implícitas corresponden al parafraseo, la repetición de la construcción errada, la solicitud de clarificación, entre otras. 
Por el contrario, cuando la corrección tiene un carácter explícito, el instructor indica que existe un error y provee una explicación y/o el uso correcto de la estructura. Esta corrección se proporciona mediante técnicas tales como la entrega de claves metalingüísticas, la corrección explícita, la corrección explícita junto con la provisión de información metalingüística, la elicitación, entre otras (Ellis, Loewen y Erlam 2006; Lyster y otros 2013).

\section{Figura 1. Feedback correctivo oral}

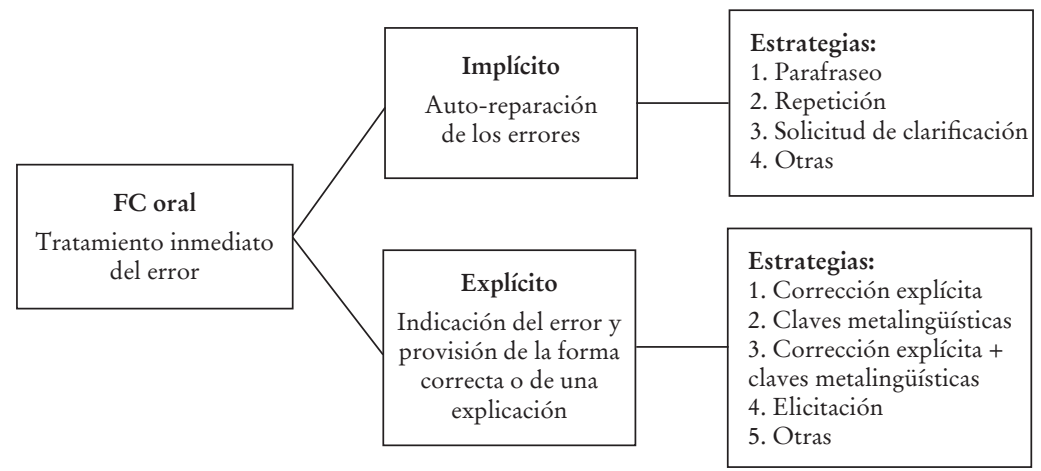

En relación con la efectividad del FC oral para la promoción de la precisión gramatical, diversos investigadores sostienen que este tipo de corrección posee un efecto positivo para el mejoramiento del uso de ciertas formas lingüísticas (McDonough 2006; Sheen 2010, 2011; Saito, en prensa). A pesar de ello, se ha reparado en la carga cognitiva que significa esta estrategia entre quienes reciben la corrección en la medida en que la reparación requiere de una comparación en línea del output y de la evidencia negativa; es decir, el aprendiz debe atender, en forma simultánea, a su producción y a la corrección.

Otro elemento que es necesario considerar en el ámbito del FC oral corresponde al número de aprendices que deben ser corregidos. Este aspecto cobra mayor sentido en contextos de instrucción formal donde, en muchas ocasiones, "oral interactions do not appear to be highly promoted” (Muñoz, Chandía y Véliz 2013: 65). 
Por lo anterior, se ha propuesto que el feedback correctivo escrito (FCE) constituiría un tratamiento del error tanto o más efectivo que el FC oral, puesto que las particularidades propias de la tarea escrita reducirían la exigencia cognitiva impuesta por el FC oral en la medida en que los estudiantes tienen un periodo más extenso para comparar entre su realización lingüística imprecisa y el feedback recibido. Lo anterior basado en el hecho de que la carga cognitiva que emerge en toda situación de corrección es menor en un contexto de escritura, cuestión que resultaría propicia para el progreso de la IL de los aprendices (Van Beuningen 2010, Sheen 2011).

\subsubsection{El feedback correctivo escrito}

El FCE, conocido también con el nombre de "corrección gramatical” o "corrección del error" (Ferris 2012), corresponde a una reacción frente a las desviaciones lingüísticas presentes en las producciones escritas de los estudiantes (Bitchener y Ferris 2012). Este tipo de reparación ha sido considerada una contribución significativa para el desarrollo de la habilidad escrita (Sheen 2010) y, por ello, ha sido utilizada por profesores de escritura en L2 con el objetivo de abordar diversos problemas que emergen en el desarrollo de la escritura, tales como la calidad textual, la precisión gramatical, la sintaxis, entre otros (Ellis, Sheen, Murakami y Takashima 2008; Sheen 2010). Este primer enfoque dio paso a una consideración de la escritura como un medio de producción lingüística que asiste el aprendizaje (“writing to learn”), razón por la que diversos investigadores en ASL han estudiado la efectividad de esta técnica de tratamiento del error en la reparación de ciertas imprecisiones lingüísticas para facilitar el desarrollo de la IL (Sheen 2010).

Lo anterior ha suscitado la creación de diversas tipologías acerca de las técnicas correctivas que pueden emplearse al momento de proporcionar el feedback en la escritura (v. Ellis 2009b, Sheen 2011). Estas clasificaciones permiten abordar con rigurosidad el tratamiento del error e identificar cuál o cuáles son las estrategias más efectivas. 
A modo general, las técnicas correctivas pueden clasificarse de acuerdo con "a) el tipo de técnica utilizada y b) el foco adoptado en la corrección" (Muñoz 2017: 279), tal como se representa a continuación:

Figura 2. Feedback correctivo escrito

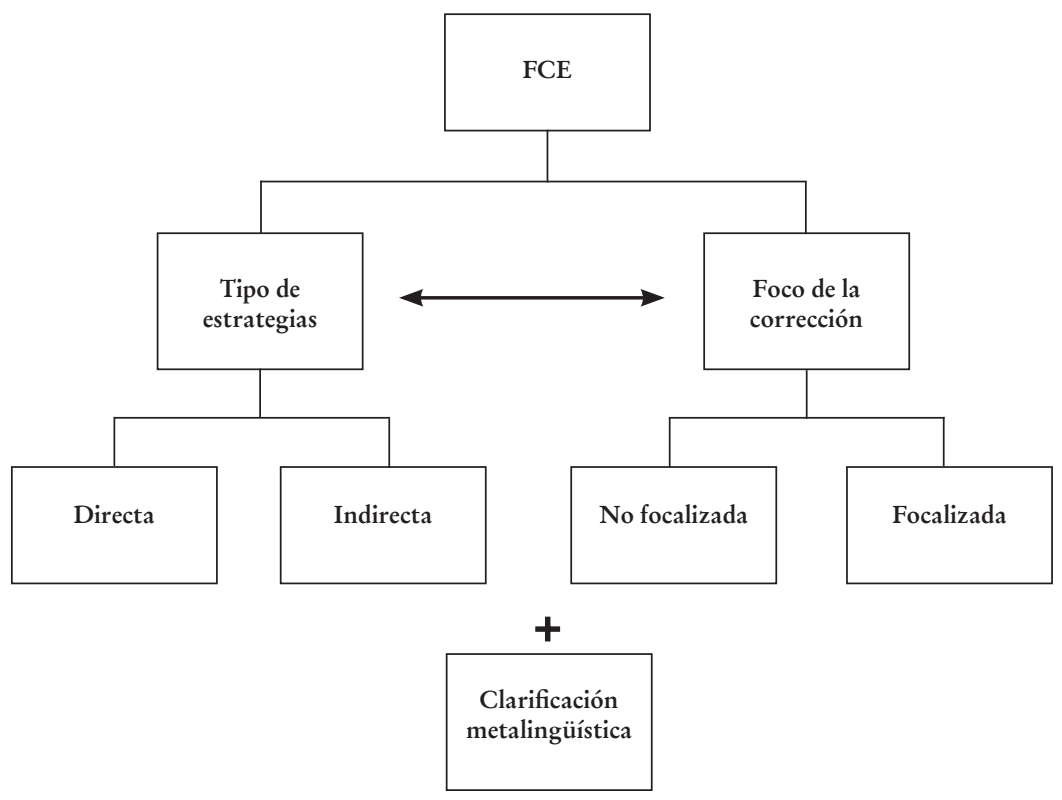

En cuanto al tipo de técnicas utilizadas para entregar retroalimentación, es posible señalar que el FCE puede ser básicamente a) directo o b) indirecto. El primero proporciona el uso correcto de la forma, como se aprecia en el siguiente ejemplo:

\section{takes}

She take a shower every morning. 
En tanto, el segundo solo proporciona alguna indicación de la existencia de una imprecisión y promueve la auto-reparación por parte del estudiante.

\section{He play tennis every Sunday.}

Además, cada una de estas técnicas puede incorporar una clarificación metalingüística para promover la reflexión metacognitiva respecto de las reglas de uso preciso, explicación que puede proporcionarse "mediante la utilización de códigos de errores, claves metalingüísticas o explicación gramatical” (Muñoz y Ferreira 2017: 77), la que puede proporcionarse en lengua materna o LE, dependiendo del nivel de proficiencia lingüística de los aprendices. Un ejemplo de esto se presenta a continuación:

\section{writes (1)}

Lisa rite beautiful poems.

(1) Remember that verbs in simple present tense add an " $\mathrm{s}$ " in third person singular.

En relación al foco del tratamiento, el FCE puede ser:

a) No focalizado, abordando todas las imprecisiones que surgen en el texto:

\section{Mary drink cofee every mornin.}

b) Focalizado, centrando la corrección en una o un número reducido de formas:

\section{Peter speak for languages.}


Bitchener y Storch (2016) señalan que, en el ámbito educativo, algunos profesores corrigen todos los errores del texto, puesto que esta forma de tratamiento sería la más beneficiosa; en tanto, otros creen que apuntar el tratamiento a solo algunas categorías de errores facilitaría el desarrollo de la L2, especialmente entre estudiantes con niveles básicos de competencia lingüística, ya que esto reduciría la carga cognitiva y, por consiguiente, aseguraría el nivel de atención requerido para procesar la corrección y realizar una adecuada modificación. Si bien esta última apreciación pareciera una razón consistente para llevar a cabo un tratamiento intensivo, los resultados respecto a la superioridad del feedback focalizado por sobre el no focalizado distan de ser concluyentes debido al limitado número de investigaciones al respecto y a los resultados discordantes (Frear y Chiu 2015).

Ahora bien, desde un punto de vista teórico, el tratamiento del error no siempre ha sido un elemento abordado de manera manifiesta por las distintas corrientes que intentan explicar el proceso de adquisición de una L2; sin embargo, al estudiar los lineamientos de cada una de estas teorías, es posible argumentar que la consideración del error y su corrección ha experimentado una valoración más bien polarizada, tal como los resultados de las investigaciones realizadas hasta ahora. El siguiente apartado aborda el lugar del tratamiento correctivo en algunas de las teorías más destacadas de ASL.

\subsection{La consideración del tratamiento del error de acuerdo con algunas teorías de ASL}

Littlewood (2004) sostiene que las perspectivas teóricas en ASL identifican distintos factores como los responsables del proceso de adquisición, cuestión que explicaría la divergencia que se observa al analizar la consideración de la importancia el error y su tratamiento bajo tales lineamientos teóricos. Si bien, no todas las corrientes que intentan explicar la ASL abordan de manera explícita el rol del tratamiento correctivo, al analizar sus postulados es posible identificar el valor que dicha acción posee en la promoción de la adquisición lingüística de los aprendices de una L2. 
Desde la perspectiva conductista, se postula que un individuo adquiere la lengua a través de la repetición o imitación de los enunciados a los que está expuesto. Esta acción es reforzada positiva o negativamente con el objetivo de ayudar al aprendiz a confirmar la exactitud de sus producciones lingüísticas o a rectificar las desviaciones o imprecisiones. Esto solo es posible gracias a la provisión de feedback o retroalimentación (Ellis 1997). Por consiguiente, bajo las orientaciones de esta corriente teórica, el tratamiento del error representa un elemento fundamental que sustenta la ejecución de las modificaciones necesarias para adquirir el hábito de uso correcto de nuevo código o, en otras palabras, para promover el uso preciso de la lengua en estudio (Van Patten y Benati 2010).

Contrariamente, las corrientes innatistas postulan que el lenguaje se desarrolla como cualquier otra función del ser humano (Chomsky 1965, O’Grady y Cho 2001) y, por esta razón, la enseñanza y la provisión de evidencia positiva o negativa sobre el uso de la lengua no tienen mayor influencia en el proceso de adquisición (Krashen 1982, Schwartz 1993) y, si la tuviesen, solo sería a nivel superficial. En otras palabras, la enseñanza y el tratamiento del error podrían modificar únicamente el conocimiento explícito de la lengua, pero no el conocimiento implícito de esta (Truscott 1998).

Una postura menos polarizada es la que deriva de las perspectivas cognitivas. Estas postulan que, si bien algunos lineamientos innatistas parecen pertinentes para explicar la adquisición de la lengua materna, estos no son suficientes para comprender el aprendizaje de una L2, suposición que conlleva la participación de elementos intervinientes en el proceso de adquisición. Esta postura asemeja la mente humana a un ordenador, ya que el aprendizaje implica almacenar y recuperar información; es decir, el procesamiento de diversas sub-habilidades que se reestructuran a medida que el sujeto avanza en la construcción de su IL. McLaughlin (1990) postula que las sub-habilidades pueden incorporarse a través de una práctica controlada que permita la reestructuración continua de la IL del estudiante y, de esta manera, se puede automatizar el conocimiento que será almacenado en su memoria a largo plazo. En este 
escenario, el tratamiento del error pudiese favorecer la automatización del nuevo código, puesto que intenciona un procesamiento controlado de las desviaciones lingüísticas apuntadas, promoviendo de esta forma su uso preciso y su incorporación al sistema interlingüístico de los aprendices.

Otro elemento que emerge bajo esta corriente teórica corresponde a la existencia de un orden natural de adquisición (Pienemann 1989, 1998); esto es, la incorporación de un nuevo código lingüístico que seguiría un trayecto rígido e invariable; por esta razón, su enseñanza debe apuntar al nivel de adquisición adecuado, ya que el aprendiente solo puede interiorizar aquellas estructuras lingüísticas que es capaz de procesar. En este escenario, es posible suponer que el tratamiento del error sería efectivo cuando está dirigido a aquellas formas gramaticales que los aprendices están preparados para incorporar a su IL (Bitchener y Ferris 2012, Muñoz y Sáez 2019).

Por su parte, Schmidt (1990, 2001, 2010), en su Hipótesis del Noticing, postula que es fundamental "notar" ciertas particularidades de las construcciones lingüísticas, puesto que no es posible aprender aquello que no es procesado de manera consciente; es decir, el aprendizaje ocurriría como un efecto secundario del proceso de atención. Por esta razón, la atención debe ser focalizada en aquellos ámbitos lingüísticos que se quieran desarrollar (Schmidt 2010). Así, de acuerdo con estas presunciones, el tratamiento del error actuaría como una estrategia que facilitaría que los aprendices atiendan a determinadas formas lingüísticas, potenciando esto la capacidad de los estudiantes de "notar" sus inconsistencias, procesarlas e incorporarlas en su construcción lingüística (Mackey 2006).

Ahora bien, en el marco de las corrientes interaccionistas, el tratamiento del error adquiere gran relevancia, pues representa un elemento favorecedor de la integración del nuevo código lingüístico. En el caso de la Hipótesis de la Interacción (Long 1996), que destaca la importancia de la negociación del significado como factor indispensable para la adquisición, el tratamiento del error posee un impacto muy relevante para el desarrollo del sistema interlingüístico del estudiante (Mackey y Oliver 2002). Por una parte, constituye 
una fuente de provisión de input lingüístico y, por otra, permite focalizar la atención del sujeto en aquellas desviaciones fonéticas, léxicas y morfosintácticas de la L2, resguardando a su vez el uso comunicativo de la lengua en estudio (Long 1996, Long y Robinson 1998). De acuerdo con Long (1996: 414), la corrección del error "obtained during negotiation work or elsewhere may be facilitative of L2 development”. Por su parte, la hipótesis del output (Swain 1985) también enfatiza la significancia del uso productivo de la lengua para la construcción de la IL del aprendiz, en el entendido de que la producción lingüística demanda un procesamiento gramatical profundo que facilita identificar las deficiencias en la lengua en estudio y comprobar las propias hipótesis de uso. En este contexto, el papel del tratamiento del error adquiere gran relevancia debido a que provee información acerca de la calidad de la producción lingüística, acción que conlleva la confirmación o modificación del output (Swain 1993, Pica 1996, McDonough 2005).

Por último, la Teoría Sociocultural (Vygotsky 1978) establece que el lenguaje se desarrolla producto del proceso de interacción social experimentado por el sujeto y que la internalización de las competencias lingüísticas corresponde a un proceso gradual que demanda, en un principio, cierta orientación y regulación externa (Ortega 2011); es decir, la adquisición se lograría en colaboración con otros, ya que esto permitiría el avance del desarrollo existente a uno potencial. Bajo estos lineamientos, diversos estudios han establecido que el tratamiento del error constituye una herramienta que asiste la construcción del andamiaje lingüístico debido a que sustenta la orientación respecto del uso preciso de la lengua, permitiendo a los aprendices conseguir un control y una autorregulación mayor en la L2 (Rassaei 2014); en otras palabras, la corrección del error impulsa el avance desde el nivel lingüístico actual al próximo nivel potencial (Lantolf y Thorne 2007, Pawlak 2014); es decir, potencia la Zona de Desarrollo Próximo. 


\section{Conclusiones}

El análisis teórico presentado aborda el trayecto que ha experimentado la consideración y tratamiento del error en la adquisición de una segunda lengua y el lugar de esta estrategia en diversas corrientes teóricas que buscan explicar dicho proceso. Si bien en un inicio, el error correspondía a un elemento indeseado que debía ser anulado del proceso de aprendizaje, esta visión dio paso a una atención al error como un factor de input respecto de la forma en que un aprendiz incorpora el nuevo código y del estadio y evolución de dicho proceso de aprendizaje (Corder 1981). El desarrollo de las perspectivas teóricas interaccionistas ha sustentado esta consideración de las desviaciones lingüísticas e, incluso, algunas de ellas abordan de manera explícita el papel de la corrección en la promoción lingüística de los aprendices.

No obstante, los dilemas introducidos por Hendrickson (1978) distan aún de ser resueltos debido a la heterogeneidad de los resultados de los estudios empíricos realizados. Probablemente, esto se deba a factores tales como los tipos de muestras incorporadas: cuestiones como la edad, los niveles de proficiencia lingüística, las motivaciones que subyacen el acercamiento a la L2, entre otros aspectos, representan variables que deben ser consideradas al momento de realizar presunciones y generalizaciones. Además, elementos como el tipo de error apuntado, las estrategias de corrección utilizadas y los contextos de aprendizaje estudiados también pudiesen dificultar la consecución de resoluciones precisas, impidiendo esto la extensión de los resultados a otros escenarios de instrucción; especialmente, a escenarios de enseñanza formal de lenguas donde la corrección del error constituye parte consustancial del proceso de enseñanza-aprendizaje.

En síntesis, es posible afirmar que la consideración del error y su tratamiento continúan siendo elementos que requieren de mayor respuesta y, por lo tanto, es necesario el desarrollo de más y variados estudios al respecto. Si bien el error y su corrección son elementos que forman parte de todo proceso de aprendizaje lingüístico, 
pareciera ser que en escenarios de enseñanza formal de segundas lenguas se torna esencial contar con antecedentes precisos referentes a qué errores responden positivamente a la corrección, cuál es el momento más propicio para corregirlos, quién debería estar a cargo de dicho proceso y cuáles son las técnicas más efectivas; todo ello, en pos del mejoramiento del proceso de adquisición lingüística.

\section{Referencias bibliográficas}

Amrhein, Hannah y Hossein NAssaji

2010 "Written corrective feedback: What do students and teachers think is right and why?". Canadian Journal of Applied Linguistics. 13, 2, 95-127.

Berg, E. Cathrine

1999 "The effects of trained peer response on ESL students' revision types and writing quality". Journal of Second Language Writing. 8, 3, 215-241.

BITCHENER, John

2008 "Evidence in support of written corrective feedback". Journal of Second Language Writing. 17, 102-118. <https://doi. org/10.1016/j.jslw.2007.11.004>

BitCHENER, John

2012 "A reflection on 'the language learning potential' of written CF”. Journal of Second Language Writing. 21, 348-363. $<$ https://doi.org/10.1016/j.jslw.2012.09.006>

Bitchener, John y Dana Ferris

2012 Written corrective feedback in second language acquisition and writing. Nueva York: Routledge.

Bitchener, John y Ute KNOCH

2009 "The value of a focused approach to written corrective feedback”. ELT Journal. 63, 204-211. <https://doi. org/10.1093/elt/ccn043>

Bitchener, John y Neomy STORCH

2016 Written corrective feedback for L2 development. UK: Multilingual Matters. 
Bitchener, John; Stuart Young; y Denise Cameron

2005 "The effect of different types of corrective feedback on ESL student writing". Journal of Second Language Writing. 14, 191-205. <https://doi.org/10.1016/j.jslw.2005.08.001>

Brown, H. Douglas

2007 Principles of language learning and teaching. Nueva York: Pearson Education Inc.

BRown, Alan

2009 'Students' and teachers' perceptions of effective foreign language teaching: A comparison of ideals". Modern Language Journal. 93, 46-60. <https://doi.org/10.1111 /j.1540-4781.2009.00827.x>

Burt, Marina

1975 "Error analysis in the adult EFL classroom". TESOL Quarterly. 9, 53-63.<https://doi.org/10.2307/3586012>

Chomsky, Noam

1965 Aspects of the theory of syntax. Cambridge, MA: MIT Press.

Corder, S. Pit

1967 “The significance of learners' errors”. International Review of Applied Linguistics. 5, 161-170.

Corder, S. Pit

1973 Introducing applied linguistics. Londres: Penguin Books.

CORDER, S. Pit

1981 Error analysis and interlanguage. Oxford: Oxford University Press.

CRYsTAL, David

2008 A dictionary of linguistics and phonetics. Oxford, UK: Blackwell Publishing.

Chaudron, Craig

1977 "A descriptive model of discourse in the corrective treatment of learners' errors”. Language learning. 27, 1, 29-46. <https:// doi.org/10.1111/j.1467-1770.1977.tb00290.x>

Chaudron, Craig

1988 Second language classrooms: Research on teaching and learning. Cambridge, UK: Cambridge University Press. 
DEKEYSER, Robert

2009 "Cognitive psychological processes in second language learning". En Handbook of second language teaching. Eds., Michael Long y Catherine Doughty. Oxford: Wiley Blackwell, 119-138.

ELLIS, Rod

1994 The study of second language acquisition. Oxford: Oxford University Press.

ELLIS, Rod

1997 Second language acquisition. Oxford: Oxford University Press.

ELLIS, Rod 2006

"Researching the effects of form-focused instruction on L2 acquisition". AILA Review. 19, 18-41. <https://doi. org/10.1075/aila.19.04ell>

ELLIS, Rod $2009 a$

"Corrective feedback and teacher development". L2 Journal. 1, 1, 3-18.

ELLIS, Rod

2009b "A typology of written corrective feedback types". ELT Journal. 62, 2, 97-107.

ElLIS, Rod; Shawn Loewen; y Rosemary ERLAM

2006 "Implicit and explicit corrective feedback and the acquisition of L2 grammar". Studies in Second Language Acquisition. 28, 2, 339-368.

Ellis, Rod; Young Sheen; Mihoko Murakami; y Hide Takashima 2008 "The effects of focused and unfocused written corrective feedback in an English as a foreign language context". System. 36, 353-371. <https://doi.org/10.1016/j.system.2008.02.001>

FERRIS, Dana

1999 "The case for grammar correction in L2 writing classes: A response to Truscott (1996)". Journal of Second Language Writing. 8, 1-10. 
FERRIS, Dana

2004 "The "grammar correction" debate in L2 writing: Where are we, and where do we go from here? (And what do we do in the meantime ...?)". Journal of Second Language Writing. 13, 49-62. <https://doi.org/10.1016/j.jslw.2004.04.005>

FERRIS, Dana

2011 Treatment of error in second language writing. EE.UU.: The University of Michigan Press.

FERris, Dana

2012 "Written corrective feedback in second language acquisition and writing studies". Language Teaching. 45, 4, 446-459. $<$ https://doi.org/10.1017/S0261444812000250>

Frear, David y Yi-Hui ChIU

2015 "The effect of focused and unfocused indirect written corrective feedback on EFL learners' accuracy in new pieces of writing". System. 53, 24-34. <https://doi.org/10.1016/j. system.2015.06.006>

Ghani, Mamuna y Tahira Asgher

2012 "Effects of teacher and peer feedback on students' writing at secondary level". Journal of Educational Research. 15, 2, 8497.

GuÉNETTE, Danielle

2012 "The pedagogy of error correction: Surviving the written corrective feedback challenge". TESL Canada Journal. 30, 1, HARLEY, Birgit 117-126. <https://doi.org/10.18806/tesl.v30i1.1129>

1993 "Instructional strategies and SLA in early French immersion". Studies in Second Language Acquisition. 15, 2, 245-260. $<$ https://doi.org/10.1017/S0272263100011980>

Hattie, John y Helen Timperley

2007 "The power of feedback". Review of educational research. 77, 1, 81-112. <https://doi.org/10.3102/003465430298487>

Hendrickson, James

1978 "Error correction in foreign language teaching: Recent research and practice". The Modern Language Journal. 62, 387-398. <https://doi.org/10.1111/j.1540-4781.1978.tb02409. 
Hyland, Ken y Fiona Hyland

2006 Feedback in second language writing. Cambridge: Cambridge University Press.

Jean, Gladys y Daphnée Simard

2011 "Grammar teaching and learning in L2: Necessary, but boring?”. Foreign Language Annals. 44, 467-494. <https://doi. org/10.1111/j.1944-9720.2011.01143.x>

Krashen, Stephen

1982 Principles and practice in second language acquisition. Oxford: Pergamon Press.

LANTOLF, James y Steven Thorne

2007 Sociocultural theory and second language learning. En Theories in second language acquisition: An introduction. Eds., Bill Van Patten y Jessica Williams. Mahwah, Nueva Jersey: Lawrence Erlbaum Associates, Inc., 197-220.

LI, Shaofeng

2017 "Student and teacher beliefs and attitude about oral corrective feedback". En Corrective feedback in second language teaching and learning: Research, Theory, applictions, implications. Eds., Hossein Nassaji y Eva Kartchava. Nueva York: Routledge. 143-157.

Lightbown, Patsy

1998 "The importance of timing in focus on form". En Focus on form in classroom second language acquisition. Eds., Catherine Doughty y Jessica Williams. Nueva York: Cambridge University Press, 177-196.

Lightbown, Patsy y Nina Spada

2006 How languages are learned. Oxford: Oxford University Press.

LiTTLEWOOD, William

2004 "Second language learning". En The handbook of applied linguistics. Eds., Alan Davies y Catherine Elder. Oxford: Blackwell Publishing Ltd., 501-524.

LOCHTMAn, Katja

2002 "Oral corrective feedback in the foreign language classroom: How it affects interaction in analytic foreign language 
teaching". International Journal of Educational Research. 37, 271-283. <https://doi.org/10.1016/S0883-0355(03)00005-3>

LonG, Michael

1996 "The role of the linguistic environment in second language acquisition". En Handbook of second language acquisition. Eds., William Ritchie y Tej Bhatia. Nueva York: Academic Press, 413-468.

Long, Michael y Peter Robinson

1998 "Focus on form: Theory, research and practice". En Focus on form in classroom second language acquisition. Eds., Catherine Doughty y Jessica Williams. Nueva York: Cambridge University Press, 15-41.

Loewen, Shawn

2012 "The role of feedback". En The Routledge handbook of second language acquisition. Eds., Susan Gass y Alison Mackey. Londres: Routledge, 24-40.

Lyster, Roy y Leila RANTA

1997 "Corrective feedback and learner uptake. Negotiation of form in communicative classrooms". Studies in Second Language Acquisition. 20, 37-66.

Lyster, Roy; Kazuya Saito; y Masatoshi Sato

2013 "Oral corrective feedback in second language classrooms". Language Teaching. 36, 1, 1-40.

Mackey, Alison

2006 "Feedback, noticing and instructed second language learning". Applied Linguistics. 27, 405-530. <https://doi.org/10.1093/ applin/ami051>

Mackey, Alison y Rhonda Oliver

2002 "Interactional feedback and children's L2 development". System. 30, 459-477. <https://doi.org/10.1016/S0346251X(02)00049-0>

Martínez, Juan de Dios

2013 "An investigation into how EFL learners emotionally respond to teachers' oral corrective feedback". Colombian Applied Linguistics Journal. 15, 29, 265-278. < https://doi.org/10.14483/ udistrital.jour.calj.2013.2.a08> 
McDonough, Kim

2005 "Negative feedback and learners' response on ESL question development”. Studies in Second Language Acquisition. 27, 1, 79-103.

McDonough, Kim

2006 "Interaction and syntactic priming: English L2 speakers' production of dative constructions". Studies in Second Language Acquisition. 28, 179-207.

McLaughlin, Barry

1990 "Restructuring”. Applied Linguistics. 11,2, 113-128. <https:// doi.org/10.1093/applin/11.2.113>

Miao, Yang; Richard Badger; y Yu ZheN

2006 "A comparative study of peer and teacher feedback in a Chinese EFL writing class”. Journal of Second Language Writing. 15, 179-200. <https://doi.org/10.1016/j.jslw.2006.09.004>

MuÑOZ, Belén

2017 "Contribución del feedback correctivo escrito indirecto en el aprendizaje del morfema -s de verbos en inglés en tercera persona singular, en estudiantes de enseñanza básica”. Literatura y Lingüistica. 35, 275-296.

MuÑoz, Belén; Mauricio Chandia; y Mauricio Véliz

2013 "On the use of the communicative approach at school level in Hualpén: A qualitative study”. Paideia. 52, 55-70.

MuÑoz, Belén y Anita Ferreira

2017 "El feedback correctivo escrito indirecto en el aprendizaje de la forma comparativa de adjetivos en inglés”. Logos. 27, 1, 7389. <https://doi.org/10.15443/RL2706>

MuÑoz, Belén y Katia Sáez

2019 "El feedback correctivo escrito indirecto en el tratamiento de la concordancia sujeto-verbo en tercera persona singular entre estudiantes de inglés como LE”. Alpha. 49, 275-290.

O’Grady, William y Sook Cho

2001 "First language acquisition". En Contemporary linguistics: An introduction. Eds., William O'Grady, John Archibal, Mark Aronoff y Janie Rees-Miller. J. Boston: Bedford/St. Martin's, 409-448. 
Ortega, Lourdes

2011 "Second language acquisition". En The Routledge handbook of applied linguistics. Ed., Simpson. Londres: Routledge, 171184.

OrTIZ, Mabel

2016 "Uso de la retroalimentación correctiva focalizada indirecta con claves metalingüísticas en la adquisición del sufijo -s en la tercera persona del singular en inglés, en estudiantes de un programa de formación pedagógica en EFL de una universidad chilena”. Folios. 44, 127-136.

PaWlak, Miroslav

2014 Error correction in the foreign language classroom. Reconsidering the issues. Berlín: Springer-Verlag.

PicA, Teresa

1996 "The essential role of negotiation in the communicative classroom”. JALT Journal. 18, 2, 241-268.

Pienemann, Manfred

1989 “Is language teachable?". Applied Linguistics. 10,1, 52-79.

Pienemann, Manfred

1998 "Hypothesis space". En Language processing and second language acquisition: Processability theory. Amsterdam: John Benjamins, 231-330.

Pienemann, Manfred y Jörg-U Kebler

2012 "Processability theory". En The Routledge Handbook of Second Language Acquisition. Eds., Susan M. Gass y Alison Mackey. Londres y Nueva York: Routledge, 228-246. $<$ https://doi.org/10.1159/000343893>

Polio, Charlene

2012 "The acquisition of second language writing". En The Routledge Handbook of Second Language Acquisition. Eds., Susan M. Gass y Alison Mackey. Londres y Nueva York: Routledge, 319-334.

Rassaei, Ehsan

2014 "Scaffolded feedback, recasts, and L2 development: A sociocultural perspective". The Modern Language Journal. 98, 1, 417-431. <https://doi.org/10.1111/j.1540-4781.2014.12060.x> 
Rollinson, Paul

2005 "Using peer feedback in the ESL writing class". ELT Journal. 59, 1, 23-30. <https://doi.org/10.1093/elt/cci003>

SAito, Kazuya

En prensa "Corrective feedback and the development of L2 pronunciation". En The Cambridge handbook of corrective feedback in language learning and teaching. Eds., Hossein Nassaji y Eva Kartchava. Cambridge: Cambridge University Press.

SARANDi, Hedayat

2016 "Oral Corrective feedback: A question of classification and application”. Tesol Quarterly. 50, 1, 235-246.

SснміDт, Richard

1990 "The role of consciousness in second language acquisition". Applied Linguistics. 11,2, 129-158. <https://doi.org/10.1093/ applin/11.2.129>

SснміDт, Richard

2001 "Attention". En Cognition and second language instruction. Ed., Peter Robinson. Cambridge: Cambridge University Press, 3-32.

SCHмidT, Richard

2010 Attention, awareness, and individual differences in language learning. Consultado: 06 de mayo de 2020. <http:// nflrc.hawaii.edu/PDFs/SCHMIDT\%20Attention, \% 20 awareness, $\% 20$ and $\% 20$ individual $\% 20$ differences.pdf $>$

Schulz, Renate

1996 "Focus on form in the foreign languages classroom: Students' and teachers' views on error correction and the role of grammar". Foreign Language Annals. 29, 3, 343-364. <https:// doi.org/10.1111/j.1944-9720.1996.tb01247.x>

Schulz, Renate

2001

"Cultural differences in student and teacher perceptions concerning the role of grammar instruction and corrective feedback: USA-Colombia”. Modern Language Journal. 85, 2, 244-258. <https://doi.org/10.1111/0026-7902.00107> 
SCHWARTZ, Bonnie

1993 "On explicit and negative data effecting and affecting competence and linguistic behavior". Studies of Second Language Acquisition. 15, 147-163. <https://doi.org/10.1017/ S0272263100011931>

SHeEN, Youngheen

2006 Corrective feedback, individual differences, and the acquisition of English articles by second language learners. Tesis de doctorado. University of Nottingham.

SHeEN, Youngheen

2007 "The effect of focused written corrective feedback and language aptitude on ESL learners' acquisition of articles”. TESOL Quarterly. 41, 255-283. <https://doi. org/10.1002/j.1545-7249.2007.tb00059.x>

SHEEN, Youngheen

2010 "The role of oral and written corrective feedback in SLA". Studies in Second Language Acquisition. 32, 169-179.

SHEEN, Youngheen

2011 Corrective feedback, individual differences and second language learning. Nueva York: Springster.

SHEen, Youngheen y Rod Ellis

2011 "Corrective feedback in language teaching”. En Handbook of research in second language teaching and learning. Ed., Eli Hinkel. Nueva York: Routledge, 593-610.

Sheen, Youngheen; David Wright; y Anna Moldawa

2009 "Differential effects of focused and unfocused written correction on the accurate use of grammatical forms by adult ESL learners". System. 37, 556-569. < https://doi.org/10.1016/j. system.2009.09.002>

Shintani, Natsuko; Rod Ellis; y Wataro SuZUKi

2014 "Effects of written feedback and revision on learners' accuracy in using two English grammatical structures". Language Learning. 64, 1, 103-131. <https://doi.org/10.1111/ lang.12029> 
SHINTANI, Natsuko y Rod ELLIS

2013 "The comparative effect of direct written corrective feedback and metalinguistic explanation on learners' explicit and implicit knowledge of the English indefinite article”. Journal of Second Language Writing. 22, 3, 286-306. <https://doi.org/10.1016/j. jslw.2013.03.011>

SPADA, Nina

2011 "Beyond form-focused instruction: Reactions on past, present and future research". Language Teaching. 44, 225-236. $<$ https://doi.org/10.1017/S0261444810000224>

SuZuki, Wataru; Hossein Nassaji; y Konosuke Sato

2019 "The effects of feedback explicitness and type of target structure on accuracy in revision and new pieces of writing”. System. 81, 135-145. <https://doi.org/10.1016/j.system.2018.12.017>

SwaIN, Merrill

1985 "Communicative competence: Some roles of comprehensible input and comprehensible output in its development”. En Input in second language acquisition. Eds., Susan Gass y Carolyn Madden. Rowley, MA: Newbury House, 236-244.

SWAIN, Merrill

1993 "The output hypothesis: Just speaking and writing aren't enough". Canadian Modern Language. 50, 1, 158-164. $<$ https://doi.org/10.3138/cmlr.50.1.158>

TRusCOTT, John

1996 "The case against grammar correction in L2 writing classes". Language Learning. 46, 2, 327-369. <https://doi. org/10.1111/j.1467-1770.1996.tb01238.x>

TRusCOTT, John

1998 "Noticing in second language acquisition: A critical review". Second Language Research. 14,2, 103-135. <https://doi. org/10.1191/026765898674803209>

TRusCOTT, John

2001 "Selecting Errors for Selective Error Correction". Concentric. 27, 2, 93-108. 
TRUSCOTT, John

2004 "Evidence and conjecture on the effects of correction: A response to Chandler". Journal of Second Language Writing. 13, 4, 337-343. <https://doi.org/10.1016/j.jslw.2004.05.002>

TRusCOTT, John

2007 "The effect of error correction on learners' ability to write accurately”. Journal of Second Language Writing. 16, 255-272. $<$ https://doi.org/10.1016/j.jslw.2007.06.003>

TRusCOTT, John

2009 "Arguments and appearances: a response to Chandler". Journal of Second Language Writing. 18, 59-60. <https://doi. org/10.1016/j.jslw.2008.09.001>

UsHiodA, Ema y Zoltan DÖRNYEI

2012 "Motivation". En The Routledge Handbook of Second Language Acquisition. Eds., Susan M. Gass y Alison Mackey. Londres y Nueva York: Routledge, 396-419.

Van Beuningen, Catherine

2010 "Corrective feedback in L2 writing: Theoretical perspectives, empirical insights, and future directions". International Journal of English Studies. 10, 2, 1-27. <https://doi.org/10.6018/ ijes/2010/2/119171>

Van Patten, Bill y Alessandro Benatti

2010 Key terms in second language acquisition. Londres: Continuum International Publishing Group.

Villamil, Olga y María De Guerrero

1998 "Assessing the impact of peer revision on L2 writing". Applied Linguistics. 19, 4, 491-514. <https://doi.org/10.1093/ applin/19.4.491>

VyGOTSKY, Lev

1978 Mind in society: The development of higher psychological processes. Massachusetts: Harvard University Press.

Recepción: 08/06/2020

Aceptación: 12/01/2021 